\title{
Subclass of Multivalent $\beta$-Uniformly Functions with Varying Arguments
}

\author{
R. M. El-Ashwah, ${ }^{1}$ M. K. Aouf, ${ }^{2}$ A. A. M. Hassan, ${ }^{3}$ and A. H. Hassan ${ }^{3}$ \\ ${ }^{1}$ Department of Mathematics, Faculty of Science, Damietta University, New Damietta 34517, Egypt \\ ${ }^{2}$ Department of Mathematics, Faculty of Science, Mansoura University, Mansoura 33516, Egypt \\ ${ }^{3}$ Department of Mathematics, Faculty of Science, Zagazig University, Zagazig 44519, Egypt
}

Correspondence should be addressed to A. H. Hassan; alaahassan1986@yahoo.com

Received 14 August 2012; Accepted 7 October 2012

Academic Editor: Nak Cho

Copyright (c) 2013 R. M. El-Ashwah et al. This is an open access article distributed under the Creative Commons Attribution License, which permits unrestricted use, distribution, and reproduction in any medium, provided the original work is properly cited.

We introduce a class of multivalent $\beta$-uniformly functions with varying arguments, give some properties of functions in this class, and obtain sharp results including coefficient estimates and distortion theorems. Finally, we give the extreme points for this class.

\section{Introduction}

Let $A(p)$ denote the class of functions of the following form:

$$
f(z)=z^{p}+\sum_{k=p+1}^{\infty} a_{k} z^{k} \quad(p \in \mathbb{N}=\{1,2,3, \ldots\}),
$$

which are analytic and $p$-valent in the open unit $\operatorname{disc} U=\{z \in$ $\mathbb{C}:|z|<1\}$. A function $f(z) \in A(p)$ is said to be $p$-valent starlike of order $\alpha$ if it satisfies the following inequality:

$$
\operatorname{Re}\left\{\frac{z f^{\prime}(z)}{f(z)}\right\}>\alpha \quad(0 \leq \alpha<p ; p \in \mathbb{N} ; z \in U)
$$

We denote by $S_{p}(\alpha)$ the class of all $p$-valent starlike functions of order $\alpha$. Also a function $f(z) \in A(p)$ is said to be $p$-valent convex of order $\alpha$, if it satisfies the following inequality:

$$
\operatorname{Re}\left\{1+\frac{z f^{\prime \prime}(z)}{f^{\prime}(z)}\right\}>\alpha \quad(0 \leq \alpha<p ; p \in \mathbb{N} ; z \in U)
$$

We denote by $K_{p}(\alpha)$ the class of all $p$-valently convex functions of order $\alpha$. The classes $S_{p}(\alpha)$ and $K_{p}(\alpha)$ were introduced by Patil and Thakare [1] and Owa [2]. Further from (2) and (3), we can see that

$$
f(z) \in K_{p}(\alpha) \Longleftrightarrow \frac{z f^{\prime}(z)}{p} \in S_{p}(\alpha) \quad(0 \leq \alpha<p ; p \in \mathbb{N}) .
$$

Also for a function $f(z) \in A(p)$, the differential operator $D_{p}^{n} f(z)$ was defined by Kamali and Orhan [3], Orhan and Kiziltunç [4], and Aouf and Mostafa [5] as follows:

$$
\begin{gathered}
D_{p}^{0} f(z)=f(z), \\
D_{p}^{1} f(z)=D_{p} f(z)=\frac{z}{p} f^{\prime}(z)=z^{p}+\sum_{k=p+1}^{\infty}\left(\frac{k}{p}\right) a_{k} z^{k}, \\
D_{p}^{2} f(z)=D_{p}\left(D_{p}^{1} f(z)\right)=z^{p}+\sum_{k=p+1}^{\infty}\left(\frac{k}{p}\right)^{2} a_{k} z^{k},
\end{gathered}
$$

and (in general)

$$
\begin{gathered}
D_{p}^{n} f(z)=z^{p}+\sum_{k=p+1}^{\infty}\left(\frac{k}{p}\right)^{n} a_{k} z^{k} \\
\left(p \in \mathbb{N} ; n \in \mathbb{N}_{0}=\mathbb{N} \cup\{0\}\right) .
\end{gathered}
$$


We note that putting $p=1$ in (6), we have the operator $D_{1}^{n}=$ $D^{n}$ which was introduced by Sălăgean [6].

In [7], Eker and Şeker introduced the class $N_{p}(m, n ; \alpha, \beta)$ as follows.

Definition 1. For $0 \leq \alpha<1, \beta \geq 0, \quad m \in \mathbb{N}, n \in \mathbb{N}_{0}, m>$ $n$, and $z \in U$, let $N_{p}(m, n ; \alpha, \beta)$ be the class of functions $f(z) \in A(p)$ which satisfy

$$
\operatorname{Re}\left\{\frac{D_{p}^{m} f(z)}{D_{p}^{n} f(z)}-\alpha\right\}>\beta\left|\frac{D_{p}^{m} f(z)}{D_{p}^{n} f(z)}-1\right| .
$$

Specializing the parameters $\beta, m, n$, and $p$ we obtain the following subclasses studied by various authors:

(i) $N_{p}(1,0 ; \alpha, \beta)=U S T(\alpha, \beta, p)$ and $N_{p}(2,1 ; \alpha, \beta)=$ $U C V(\alpha, \beta, p)(0 \leq \alpha<p, \beta \geq 0, p \in \mathbb{N})$ (see Khairnar and More [8]);

(ii) $N_{p}(1,0 ; \alpha, o)=S^{*}(p, \alpha)$ and $N_{p}(2,1 ; \alpha, 0)=$ $K(p, \alpha)(0 \leq \alpha<p, p \in \mathbb{N})$ (see Patil and Thakare [1] and Owa [2]);

(iii) $N_{1}(m, n ; \alpha, \beta)=N_{m, n}(\alpha, \beta)(0 \leq \alpha<1, \beta \geq 0, m \in$ $\mathbb{N}, n \in \mathbb{N}_{0}, m>n$ ) (see Eker and Owa [9]);

(iv) $N_{1}(m, n ; \alpha, 0)=K_{m, n}(\alpha)(0 \leq \alpha<1, m \in \mathbb{N}, n \in$ $\left.\mathbb{N}_{0}, m>n\right)$ (see Eker and Owa [10]);

(v) $N_{1}(n+1, n ; \alpha, \beta)=S(n, \alpha, \beta)(0 \leq \alpha<1, \beta \geq 0, n \in$ $\mathbb{N}_{0}$ ) (see Rosy and Murugusundaramoorthy [11] and Aouf [12]);

(vi) $N_{1}(1,0 ; \alpha, \beta)=S D(\alpha, \beta)$ and $N_{1}(2,1 ; \alpha, \beta)=$ $K D(\alpha, \beta)(0 \leq \alpha<1, \beta \geq 0)$ (see Shams et al. [13] and Bharati et al. [14]).

Also we note that

$$
\begin{aligned}
N_{p}(n+1, n ; \alpha, \beta)= & N_{p}(n ; \alpha, \beta) \\
= & \left\{f \in A(p): \operatorname{Re}\left\{\frac{D_{p}^{n+1} f(z)}{D_{p}^{n} f(z)}-\alpha\right\}\right. \\
& >\beta\left|\frac{D_{p}^{n+1} f(z)}{D_{p}^{n} f(z)}-1\right| \\
& \left.\left(0 \leq \alpha<1 ; \beta \geq 0 ; n \in \mathbb{N}_{0} ; z \in U\right)\right\} .
\end{aligned}
$$

Silverman [15] defined the class of univalent functions $f(z)=$ $z+\sum_{k=2}^{\infty} a_{k} z^{k}$ for which $\arg \left(a_{k}\right)$ prescribed in such a way that $f(z)$ is univalent if and only if $f(z)$ is starlike. In this paper, we introduce the subclass of $p$-valent functions with varying arguments as follows.

Definition 2. A function $f(z)$ of the form (1) is said to be in the class $V_{p}\left(\theta_{k}\right)$ if $f \in A(p)$ and $\arg \left(a_{k}\right)=\theta_{k}$ for all $k \geq$ $p+1$. If furthermore there exists a real number $\delta$ such that $\theta_{k}+(k-p) \delta \equiv \pi(\bmod 2 \pi)$ for all $k \geq p+1$, then $f(z)$ is said to be in the class $V_{p}\left(\theta_{k}, \delta\right)$. The union of $V_{p}\left(\theta_{k}, \delta\right)$ taken over all possible sequences $\left\{\theta_{k}\right\}$ and all possible real numbers $\delta$ is denoted by $V_{p}$.

Let $V_{p}(m, n ; \alpha, \beta)$ denote the subclass of $V_{p}$ consisting of functions $f(z) \in N_{p}(m, n ; \alpha, \beta)$.

We note that $V_{1}(1,0 ; \alpha, 0)=V^{*}(\alpha)$, which was introduced and studied by Silverman [15].

Specializing the parameters $\beta, m, n$, and $p$, we obtain the following classes with varying arguments which are associated to the subclasses mentioned before:

(i) $V_{p}(1,0 ; \alpha, \beta)=\operatorname{VST}(\alpha, \beta, p)$ and $V_{p}(2,1 ; \alpha, \beta)=$ $\operatorname{VCV}(\alpha, \beta, p)(0 \leq \alpha<p, \beta \geq 0, p \in \mathbb{N})$;

(ii) $V_{p}(1,0 ; \alpha, o)=V S^{*}(p, \alpha)$ and $V_{p}(2,1 ; \alpha, 0)=$ $V K(p, \alpha)(0 \leq \alpha<p, p \in \mathbb{N})$;

(iii) $V_{1}(m, n ; \alpha, \beta)=V_{m, n}(\alpha, \beta) \quad(0 \leq \alpha<1, \beta \geq 0, m \in$ $\left.\mathbb{N}, n \in \mathbb{N}_{0}, m>n\right)$;

(iv) $V_{1}(m, n ; \alpha, 0)=V_{m, n}(\alpha) \quad(0 \leq \alpha<1, m \in \mathbb{N}, n \in$ $\left.\mathbb{N}_{0}, m>n\right)$;

(v) $V_{1}(n+1, n ; \alpha, \beta)=V(n, \alpha, \beta) \quad(0 \leq \alpha<1, \beta \geq 0, n \in$ $\mathbb{N}_{0}$;

(vi) $V_{1}(1,0 ; \alpha, \beta)=\operatorname{VSD}(\alpha, \beta)$ and $V_{1}(2,1 ; \alpha, \beta)=$ $\operatorname{VKD}(\alpha, \beta)(0 \leq \alpha<1, \beta \geq 0)$;

(vii) $V_{p}(n+1, n ; \alpha, \beta)=V_{p}(n ; \alpha, \beta)$.

In this paper, we obtain coefficient bounds for functions in the class $V_{p}(m, n ; \alpha, \beta)$, further we obtain distortion theorems and the extreme points for functions in this class.

\section{Coefficient Estimates}

Unless otherwise mentioned, we assume in the reminder of this paper that $0 \leq \alpha<1, \beta \geq 0, m \in \mathbb{N}, n \in \mathbb{N}_{0}, m>n$, and $z \in U$.

We will need the following lemma.

Lemma 3. The sufficient condition for $f(z)$ given by (1) to be in the class $N_{p}(m, n ; \alpha, \beta)$ is that

$$
\sum_{k=p+1}^{\infty}\left[(1+\beta)\left(\frac{k}{p}\right)^{m}-(\alpha+\beta)\left(\frac{k}{p}\right)^{n}\right]\left|a_{k}\right| \leq 1-\alpha .
$$

Proof. It suffices to show that inequality (7) holds true. Then it suffices to show that

$$
\beta\left|\frac{D_{p}^{m} f(z)}{D_{p}^{n} f(z)}-1\right|-\operatorname{Re}\left\{\frac{D_{p}^{m} f(z)}{D_{p}^{n} f(z)}-1\right\} \leq 1-\alpha .
$$

We have

$$
\begin{gathered}
\beta\left|\frac{D_{p}^{m} f(z)}{D_{p}^{n} f(z)}-1\right|-\operatorname{Re}\left\{\frac{D_{p}^{m} f(z)}{D_{p}^{n} f(z)}-1\right\} \\
\leq(1+\beta)\left|\frac{D_{p}^{m} f(z)}{D_{p}^{n} f(z)}-1\right|,
\end{gathered}
$$


or, equivalently,

$$
\begin{aligned}
\beta\left|\frac{D_{p}^{m} f(z)}{D_{p}^{n} f(z)}-1\right|-\operatorname{Re}\left\{\frac{D_{p}^{m} f(z)}{D_{p}^{n} f(z)}-1\right\} \\
\leq(1+\beta)\left|\frac{\sum_{k=p+1}^{\infty}\left[(k / p)^{m}-(k / p)^{n}\right] a_{k} z^{k}}{z^{p}+\sum_{k=p+1}^{\infty}(k / p)^{n} a_{k} z^{k}}\right| \\
\leq(1+\beta)\left|\frac{\sum_{k=p+1}^{\infty}\left[(k / p)^{m}-(k / p)^{n}\right] a_{k} z^{k-p}}{1+\sum_{k=p+1}^{\infty}(k / p)^{n} a_{k} z^{k-p}}\right|,
\end{aligned}
$$

then we have

$$
\begin{aligned}
& \beta\left|\frac{D_{p}^{m} f(z)}{D_{p}^{n} f(z)}-1\right|-\operatorname{Re}\left\{\frac{D_{p}^{m} f(z)}{D_{p}^{n} f(z)}-1\right\} \\
& \leq(1+\beta) \frac{\sum_{k=p+1}^{\infty}\left[(k / p)^{m}-(k / p)^{n}\right]\left|a_{k}\right||z|^{k-p}}{1-\sum_{k=p+1}^{\infty}(k / p)^{n}\left|a_{k}\right||z|^{k-p}} .
\end{aligned}
$$

Letting $z \rightarrow 1^{-}$through real values, then we have

$$
\begin{array}{r}
\beta\left|\frac{D_{p}^{m} f(z)}{D_{p}^{n} f(z)}-1\right|-\operatorname{Re}\left\{\frac{D_{p}^{m} f(z)}{D_{p}^{n} f(z)}-1\right\} \\
\leq(1+\beta) \frac{\sum_{k=p+1}^{\infty}\left[(k / p)^{m}-(k / p)^{n}\right]\left|a_{k}\right|}{1-\sum_{k=p+1}^{\infty}(k / p)^{n}\left|a_{k}\right|} .
\end{array}
$$

The last expression is bounded above by $(1-\alpha)$ if

$$
\sum_{k=p+1}^{\infty}\left[(1+\beta)\left(\frac{k}{p}\right)^{m}-(\alpha+\beta)\left(\frac{k}{p}\right)^{n}\right]\left|a_{k}\right| \leq 1-\alpha .
$$

And hence the proof of Lemma 3 is completed.

Remark 4. (i) The result obtained from Lemma 3 corrects the result obtained by Eker and Şeker [7, Theorem 1].

(ii) Putting $p=1$ in Lemma 3, we correct the result obtained by Eker and Owa [9, Theorem 2.1].

Corollary 5. The sufficient condition for $f(z)$ given by (1) to be in the class $N_{p}(n ; \alpha, \beta)$, which is defined by (8) is that

$$
\sum_{k=p+1}^{\infty}\left(\frac{k}{p}\right)^{n}\left[\frac{k}{p}(1+\beta)-(\alpha+\beta)\right]\left|a_{k}\right| \leq 1-\alpha .
$$

Theorem 6. Let $f(z)$ of the form (1), then $f(z) \in$ $V_{p}(m, n ; \alpha, \beta)$ if and only if

$$
\sum_{k=p+1}^{\infty}\left[(1+\beta)\left(\frac{k}{p}\right)^{m}-(\alpha+\beta)\left(\frac{k}{p}\right)^{n}\right]\left|a_{k}\right| \leq 1-\alpha .
$$

Proof. In view of Lemma 3 , we need only to show that function $f(z) \in V_{p}(m, n ; \alpha, \beta)$ satisfies the coefficient inequality (17). If $f(z) \in V_{p}(m, n ; \alpha, \beta)$, then from (7), we have

$$
\operatorname{Re}\left\{\frac{D_{p}^{m} f(z)}{D_{p}^{n} f(z)}-\alpha\right\}>\beta\left|\frac{D_{p}^{m} f(z)}{D_{p}^{n} f(z)}-1\right|,
$$

then we have

$$
\begin{gathered}
\operatorname{Re}\left\{\frac{(1-\alpha) z^{p}+\sum_{k=p+1}^{\infty}\left[(k / p)^{m}-\alpha(k / p)^{n}\right] a_{k} z^{k}}{z^{p}+\sum_{k=p+1}^{\infty}(k / p)^{n} a_{k} z^{k}}\right\} \\
>\beta\left|\frac{\sum_{k=p+1}^{\infty}\left[(k / p)^{m}-(k / p)^{n}\right] a_{k} z^{k}}{z^{p}+\sum_{k=p+1}^{\infty}(k / p)^{n} a_{k} z^{k}}\right|
\end{gathered}
$$

or

$$
\begin{gathered}
\operatorname{Re}\left\{\frac{(1-\alpha)+\sum_{k=p+1}^{\infty}\left[(k / p)^{m}-\alpha(k / p)^{n}\right] a_{k} z^{k-p}}{1+\sum_{k=p+1}^{\infty}(k / p)^{n} a_{k} z^{k-p}}\right\} \\
>\beta\left|\frac{\sum_{k=p+1}^{\infty}\left[(k / p)^{m}-(k / p)^{n}\right] a_{k} z^{k-p}}{1+\sum_{k=p+1}^{\infty}(k / p)^{n} a_{k} z^{k-p}}\right| .
\end{gathered}
$$

Since $f(z) \in V, f(z)$ lies in the class $V\left(\theta_{k}, \delta\right)$ for some sequence $\left\{\theta_{k}\right\}$ and a real number $\delta$ such that

$$
\theta_{k}+(k-p) \delta \equiv \pi(\bmod 2 \pi) \quad(k \geq p+1) .
$$

Set $z=r e^{i \delta}$ in (20), we get

$$
\begin{aligned}
& \frac{(1-\alpha)}{-\sum_{k=p+1}^{\infty}\left[(k / p)^{m}-\alpha(k / p)^{n}\right]\left|a_{k}\right| r^{k-p}} 1-\sum_{k=p+1}^{\infty}(k / p)^{n}\left|a_{k}\right| r^{k-p} \\
&>\beta\left[\frac{\sum_{k=p+1}^{\infty}\left[(k / p)^{m}-(k / p)^{n}\right]\left|a_{k}\right| r^{k-p}}{1-\sum_{k=p+1}^{\infty}(k / p)^{n}\left|a_{k}\right| r^{k-p}}\right] .
\end{aligned}
$$

Letting $r \rightarrow 1$, then we have the inequality (17).

Hence the proof of Theorem 6 is completed.

Corollary 7. If $f(z) \in V_{p}(m, n ; \alpha, \beta)$, then

$$
\begin{array}{r}
\left|a_{k}\right| \leq \frac{1-\alpha}{(1+\beta)(k / p)^{m}-(\alpha+\beta)(k / p)^{n}} \\
(k \geq p+1 ; p \in \mathbb{N}) .
\end{array}
$$

The result is sharp for the function $f(z)$ given by

$$
\begin{array}{r}
f(z)=z^{p}+\frac{1-\alpha}{(1+\beta)(k / p)^{m}-(\alpha+\beta)(k / p)^{n}} e^{i \theta_{k}} z^{k} \\
(k \geq p+1 ; p \in \mathbb{N} ; z \in U) .
\end{array}
$$

\section{Distortion Theorems}

Theorem 8. Let the function $f(z)$ defined by (1) be in the class $V_{p}(m, n ; \alpha, \beta)$. Then

$$
|z|^{p}-\frac{1-\alpha}{\Phi(p+1)}|z|^{p+1} \leq|f(z)| \leq|z|^{p}+\frac{1-\alpha}{\Phi(p+1)}|z|^{p+1},
$$


where

$$
\Phi(p+1)=(1+\beta)\left(\frac{p+1}{p}\right)^{m}-(\alpha+\beta)\left(\frac{p+1}{p}\right)^{n}
$$

The result is sharp.

Proof. We employ the same technique as used by Silverman [15]. In view of Theorem 6, since

$$
\Phi(k)=(1+\beta)\left(\frac{k}{p}\right)^{m}-(\alpha+\beta)\left(\frac{k}{p}\right)^{n},
$$

is an increasing function of $k(k \geq p+1 ; p \in \mathbb{N})$, we have

$$
\Phi(p+1) \sum_{k=p+1}^{\infty}\left|a_{k}\right| \leq \sum_{k=p+1}^{\infty} \Phi(k)\left|a_{k}\right| \leq 1-\alpha,
$$

that is

$$
\sum_{k=p+1}^{\infty}\left|a_{k}\right| \leq \frac{1-\alpha}{\Phi(p+1)}
$$

Thus, we have

$$
\begin{gathered}
|f(z)| \leq|z|^{p}+|z|^{p+1} \sum_{k=p+1}^{\infty}\left|a_{k}\right| \\
|f(z)| \\
\leq|z|^{p}+\frac{1-\alpha}{(1+\beta)((p+1) / p)^{m}-(\alpha+\beta)((p+1) / p)^{n}}|z|^{p+1} .
\end{gathered}
$$

Similarly, we get

$$
\begin{aligned}
|f(z)| \geq & |z|^{p}-|z|^{p+1} \sum_{k=p+1}^{\infty}\left|a_{k}\right| \\
\geq & |z|^{p}-\frac{1-\alpha}{(1+\beta)((p+1) / p)^{m}-(\alpha+\beta)((p+1) / p)^{n}} \\
& \times|z|^{p+1} .
\end{aligned}
$$

This completes the proof of Theorem 8. Finally the result is sharp for the following function:

$$
\begin{aligned}
f(z)= & z^{p}+\frac{1-\alpha}{(1+\beta)((p+1) / p)^{m}-(\alpha+\beta)((p+1) / p)^{n}} \\
& \times e^{i \theta_{p+1} z^{p+1}}
\end{aligned}
$$

at $z= \pm|z| e^{-i \theta_{p+1}}$.

Corollary 9. Under the hypotheses of Theorem 8, $f(z)$ is included in a disc with center at the origin and radius $r_{1}$ given by

$$
r_{1}=1+\frac{1-\alpha}{(1+\beta)((p+1) / p)^{m}-(\alpha+\beta)((p+1) / p)^{n}} .
$$

Theorem 10. Let the function $f(z)$ defined by (1) be in the class $V_{p}(m, n ; \alpha, \beta)$. Then

$$
\begin{aligned}
& \left\{p-\frac{(p+1)(1-\alpha)}{\Phi(p+1)}|z|\right\}|z|^{p-1} \\
& \leq\left|f^{\prime}(z)\right| \leq\left\{p+\frac{(p+1)(1-\alpha)}{\Phi(p+1)}|z|\right\}|z|^{p-1}
\end{aligned}
$$

where $\Phi(p+1)$ is defined by (26). Also the result of Theorem 10 is sharp.

Proof. Similarly for $\Phi(k)$ defined by (27) it is clear that $\Phi(k) / k$ is an increasing function of $k(k \geq p+1 ; p \in \mathbb{N})$, in view of Theorem 6 , we have

$$
\begin{aligned}
& \frac{\Phi(p+1)}{p+1} \sum_{k=p+1}^{\infty} k\left|a_{k}\right| \\
& \leq \sum_{k=p+1}^{\infty} \frac{\Phi(k)}{k} k\left|a_{k}\right| \\
& =\sum_{k=p+1}^{\infty} \Phi(k)\left|a_{k}\right| \leq 1-\alpha,
\end{aligned}
$$

that is

$$
\sum_{k=p+1}^{\infty} k\left|a_{k}\right| \leq \frac{(p+1)(1-\alpha)}{\Phi(p+1)}
$$

Thus, we have

$$
\begin{aligned}
\left|f^{\prime}(z)\right| & \leq p|z|^{p-1}+|z|^{p} \sum_{k=p+1}^{\infty} k\left|a_{k}\right| \\
& \leq\left\{p+|z| \sum_{k=p+1}^{\infty} k\left|a_{k}\right|\right\}|z|^{p-1} \\
& \leq\left\{p+\frac{(p+1)(1-\alpha)}{\Phi(p+1)}|z|\right\}|z|^{p-1} .
\end{aligned}
$$

Similarly,

$$
\begin{aligned}
\left|f^{\prime}(z)\right| & \geq p|z|^{p-1}-|z|^{p} \sum_{k=p+1}^{\infty} k\left|a_{k}\right| \\
& \geq\left\{p-|z| \sum_{k=p+1}^{\infty} k\left|a_{k}\right|\right\}|z|^{p-1} \\
& \leq\left\{p-\frac{(p+1)(1-\alpha)}{\Phi(p+1)}|z|\right\}|z|^{p-1} .
\end{aligned}
$$

Finally, we can see that the assertions of Theorem 10 are sharp for the function $f(z)$ defined by (32). This completes the proof of Theorem 10 . 
Corollary 11. Under the hypotheses of Theorem 10, $f^{\prime}(z)$ is included in a disc with center at the origin and radius $r_{2}$ given by

$$
r_{2}=p+\frac{(p+1)(1-\alpha)}{\Phi(p+1)}
$$

\section{Extreme Points}

Theorem 12. Let the function $f(z)$ defined by (1) be in the class $V_{p}(m, n ; \alpha, \beta)$, with $\arg \left(a_{k}\right)=\theta_{k}$, where $\theta_{k}+(k-p) \delta \equiv$ $\pi(\bmod 2 \pi)(k \geq p+1)$. Define

$$
\begin{gathered}
f_{p}(z)=z^{p} \\
f_{k}(z)=z^{p}+\frac{1-\alpha}{(1+\beta)(k / p)^{m}-(\alpha+\beta)(k / p)^{n}} e^{i \theta_{k}} z^{k} \\
(k \geq p+1 ; z \in U) .
\end{gathered}
$$

Then $f(z) \in V_{p}(m, n ; \alpha, \beta)$ if and only if $f(z)$ can be expressed in the form $f(z)=\sum_{k=p}^{\infty} \mu_{k} f_{k}(z)$, where $\mu_{k} \geq 0$ and $\sum_{k=p}^{\infty} \mu_{k}=$ 1 .

Proof. If $f(z)=\sum_{k=p}^{\infty} \mu_{k} f_{k}(z)$ with $\mu_{k} \geq 0$ and $\sum_{k=p}^{\infty} \mu_{k}=1$, then

$$
\begin{aligned}
\sum_{k=p+1}^{\infty} & {\left[(1+\beta)\left(\frac{k}{p}\right)^{m}-(\alpha+\beta)\left(\frac{k}{p}\right)^{n}\right] } \\
& \times \frac{1-\alpha}{(1+\beta)(k / p)^{m}-(\alpha+\beta)(k / p)^{n}} \mu_{k} \\
= & \sum_{k=p+1}^{\infty}(1-\alpha) \mu_{k}=(1-\alpha)\left(1-\mu_{p}\right) \leq(1-\alpha) .
\end{aligned}
$$

Hence, $f(z) \in V_{p}(m, n ; \alpha, \beta)$.

Conversely, let the function $f(z)$ defined by (1) be in the class $V_{p}(m, n ; \alpha, \beta)$. Define

$$
\begin{gathered}
\mu_{k}=\frac{\left[(1+\beta)(k / p)^{m}-(\alpha+\beta)(k / p)^{n}\right]}{1-\alpha}\left|a_{k}\right| \\
(k \geq p+1 ; p \in \mathbb{N}), \\
\mu_{p}=1-\sum_{k=p+1}^{\infty} \mu_{k} .
\end{gathered}
$$

From Theorem 6, $\sum_{k=p+1}^{\infty} \mu_{k} \leq 1$ and so $\mu_{p} \geq 0$. Since $\mu_{k} f_{k}(z)=\mu_{k} z^{p}+a_{k} z^{k}$, then

$$
\sum_{k=p}^{\infty} \mu_{k} f_{k}(z)=z^{p}+\sum_{k=p+1}^{\infty} a_{k} z^{k}=f(z) .
$$

This completes the proof of Theorem 12 .
Remark 13. (i) Putting $\beta=n=0$ and $p=m=1$ in all the above results, we obtain the corresponding results obtained by Silverman [15].

(ii) Putting (i) $\beta=n=0$ and $m=1$ and (ii) $\beta=$ $0, n=1$ and $m=2$ in all the above results, we obtain the corresponding results obtained by Aouf et al. [16].

(iii) Putting $m=n+1\left(n \in \mathbb{N}_{0}\right)$ in all the above results, we obtain the corresponding results of the classes $V_{p}(n ; \alpha, \beta)$, defined by (8).

\section{Acknowledgment}

The authors thank the referees for their valuable suggestions which led to improvement of this study.

\section{References}

[1] D. A. Patil and N. K. Thakare, "On convex hulls and extreme points of $p$-valent starlike and convex classes with applications," Bulletin Mathématique de la Société des Sciences Mathématiques de la République Socialiste de Roumanie, vol. 27, no. 75, pp. 145-160, 1983.

[2] S. Owa, "On certain classes of $p$-valent functions with negative coefficients," Simon Stevin, vol. 59, no. 4, pp. 385-402, 1985.

[3] M. Kamali and H. Orhan, "On a subclass of certain starlike functions with negative coefficients," Bulletin of the Korean Mathematical Society, vol. 41, no. 1, pp. 53-71, 2004.

[4] H. Orhan and H. Kiziltunç, "A generalization on subfamily of p-valent functions with negative coefficients," Applied Mathematics and Computation, vol. 155, no. 2, pp. 521-530, 2004.

[5] M. K. Aouf and A. O. Mostafa, "On a subclass of $n$ - $p$ valent prestarlike functions," Computers \& Mathematics with Applications, vol. 55, no. 4, pp. 851-861, 2008.

[6] G. Sălăgean, "Subclasses of univalent functions," in Complex Analysis-5h Romanian-Finnish Seminar, vol. 1013 of Lecture Notes in Mathematics, pp. 362-372, Springer, Berlin, Germany, 1983.

[7] S. S. Eker and B. Şeker, "On a class of multivalent functions defined by Salagean operator," General Mathematics, vol. 15, no. 3, pp. 154-163, 2007.

[8] S. M. Khairnar and M. More, "On a subclass of multivalent $\beta$-uniformly starlike and convex functions defined by a linear operator," IAENG International Journal of Applied Mathematics, vol. 39, no. 3, pp. 175-183, 2009.

[9] S. Sümer Eker and S. Owa, "Certain classes of analytic functions involving Salagean operator," Journal of Inequalities in Pure and Applied Mathematics, vol. 10, no. 1, article 22, 12 pages, 2009.

[10] S. S. Eker and S. Owa, "New applications of classes of analytic functions involving Salagean operator, Internat," in Proceedings of the Symposium on Complex Function Theory and Applications, Brasov, Romania, September 2006.

[11] T. Rosy and G. Murugusundaramoorthy, "Fractional calculus and their applications to certain subclass of uniformly convex functions," Far East Journal of Mathematical Sciences, vol. 15, no. 2, pp. 231-242, 2004.

[12] M. K. Aouf, "A subclass of uniformly convex functions with negative coefficients," Mathematica, vol. 52, no. 2, pp. 99-111, 2010.

[13] S. Shams, S. R. Kulkarni, and J. M. Jahangiri, "Classes of uniformly starlike and convex functions," International Journal of 
Mathematics and Mathematical Sciences, no. 55, pp. 2959-2961, 2004.

[14] R. Bharati, R. Parvatham, and A. Swaminathan, "On subclasses of uniformly convex functions and corresponding class of starlike functions," Tamkang Journal of Mathematics, vol. 28, no. 1, pp. 17-32, 1997.

[15] H. Silverman, "Univalent functions with varying arguments," Houston Journal of Mathematics, vol. 7, no. 2, pp. 283-287, 1981.

[16] M. K. Aouf, R. M. El-Ashwah, A. A. M. Hassan, and A. H. Hassan, "Multivalent functions with varying arguments," International Journal of Open Problems in Complex Analysis. in press. 


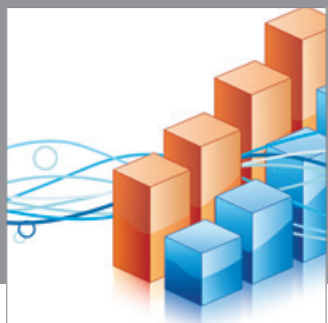

Advances in

Operations Research

mansans

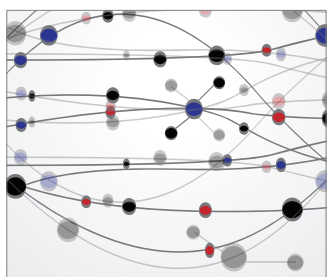

The Scientific World Journal
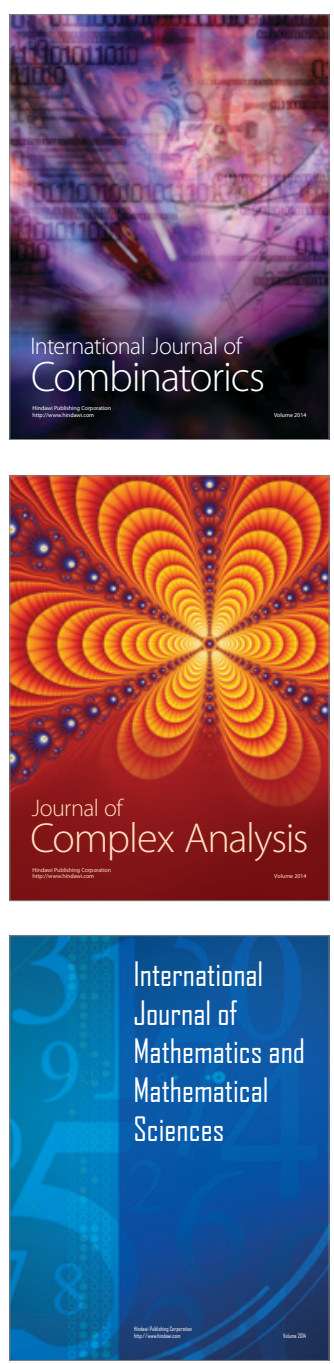
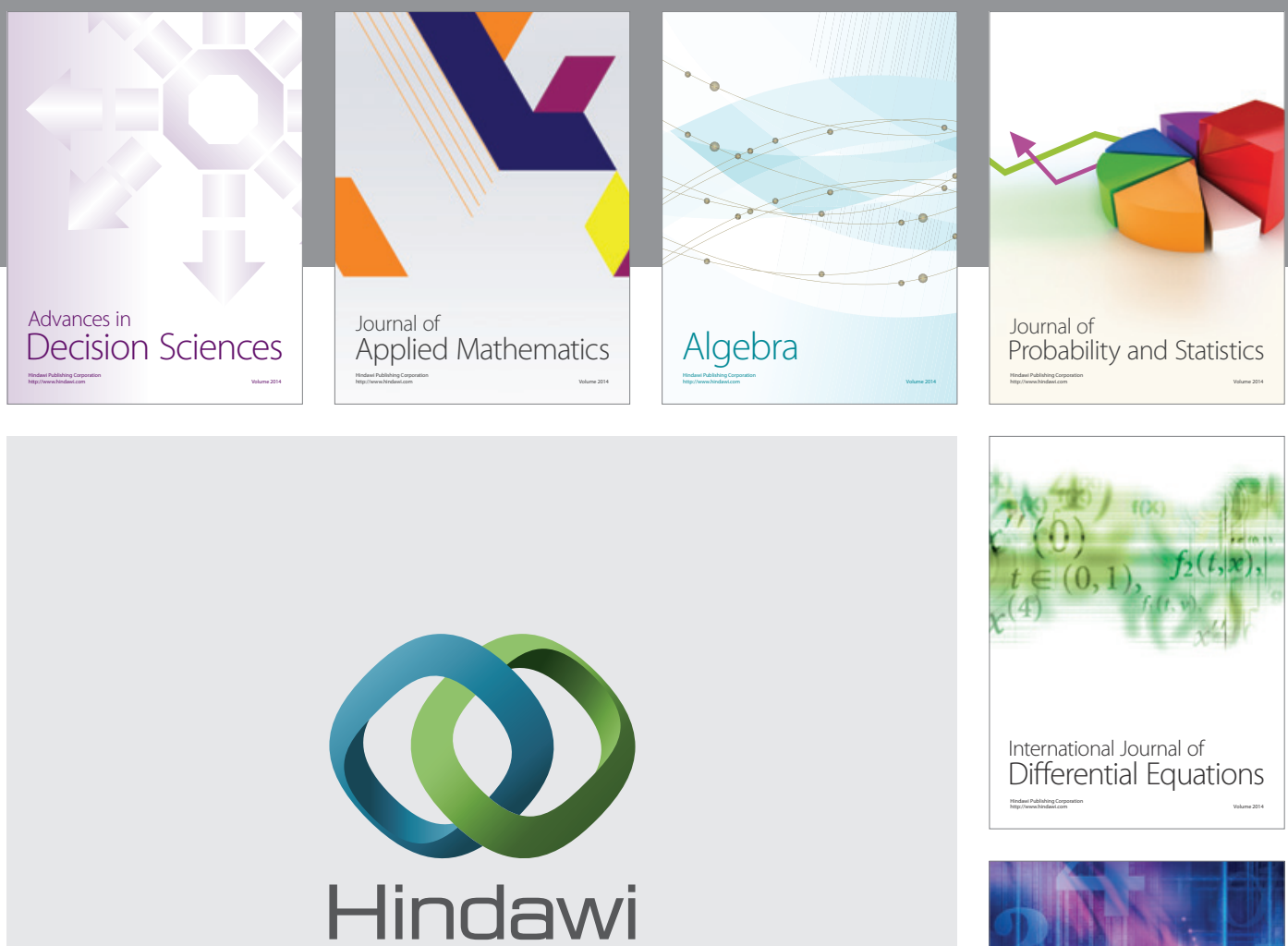

Submit your manuscripts at http://www.hindawi.com
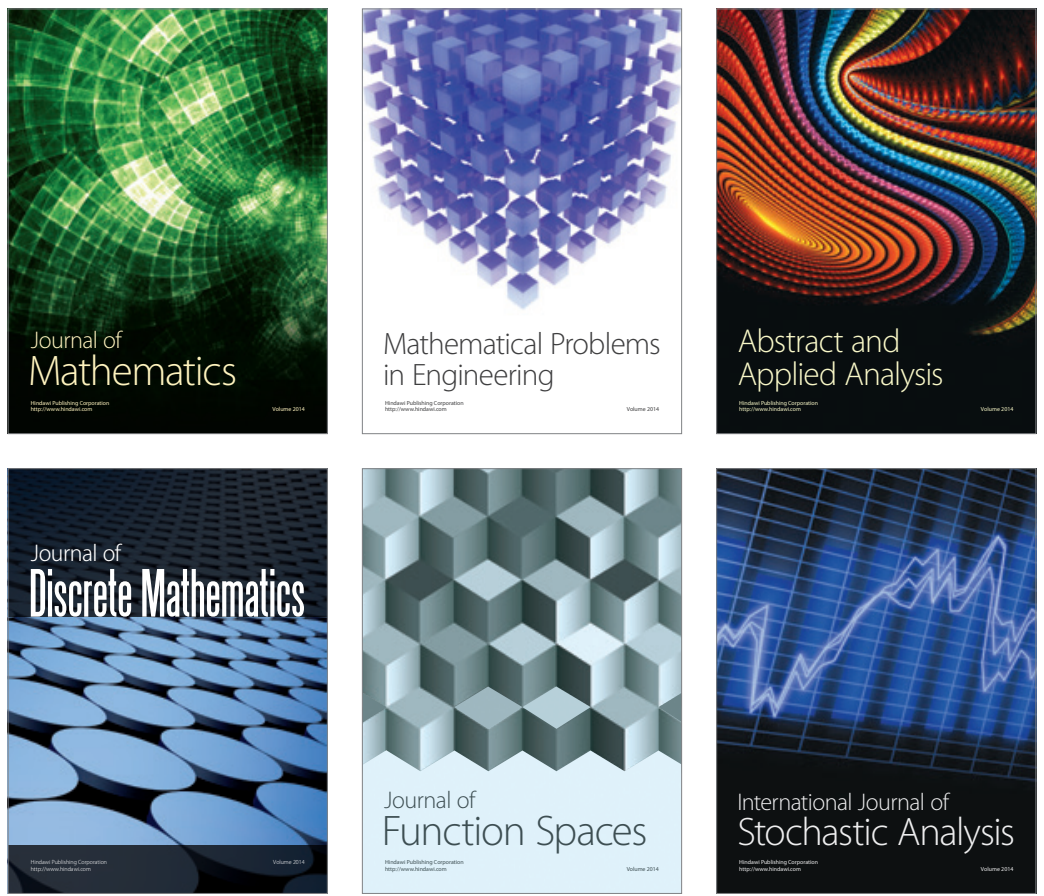

Journal of

Function Spaces

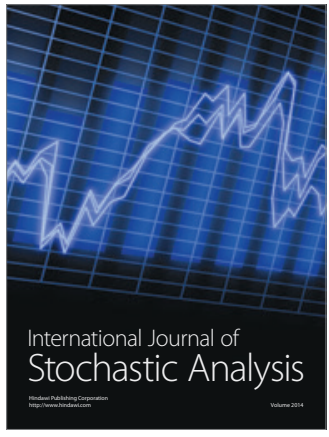

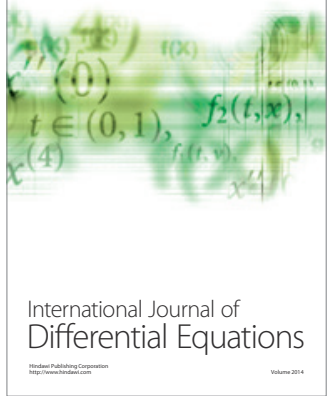
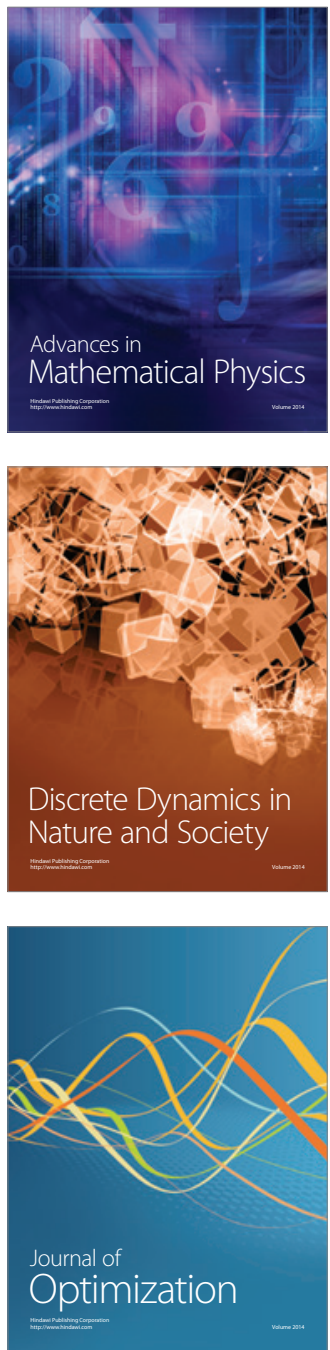